\title{
Privacidade e Segurança Digital: um estudo sobre a percepção e o comportamento dos usuários sob a perspectiva do paradoxo da privacidade
}

\author{
Hebert Junior Soares ${ }^{1}$, Nelcileno V. de S. Araújo ${ }^{1}$, Patrícia Cristiane de Souza ${ }^{1}$, \\ ${ }^{1}$ Laboratório de Ambientes Virtuais Interativos (LAVI) - Instituto de Computação - \\ Universidade Federal de Mato Grosso (UFMT) - Cuiabá, MT - Brasil \\ hebertjr20@gmail.com, nelcilenodic.ufmt.br, patricia@ic.ufmt.br
}

\begin{abstract}
The steady increase in research that discusses digital security and privacy issues and solutions is quite noticeable, as well as the increasing occurrence of worldwide cybercrime. This article discusses the relationships of perceptions and preferences in Brazil about security and privacy versus other countries in the privacy paradox bias. And it presents data analysis from a small-scale survey of users' behavior regarding digital security and privacy.
\end{abstract}

Resumo. É perceptível o aumento de pesquisas que discutem as questões de segurança digital e privacidade, bem como o aumento na ocorrência de crimes cibernéticos em todo o mundo. Nesse contexto, é proposto uma análise comparativa das percepções e preferências, no viés do paradoxo da privacidade, entre Brasil e outros países. Para tanto, tem-se a análise de dados de uma pesquisa, em pequena escala, sobre o comportamento dos usuários em relação à segurança e privacidade digital.

\section{Contextualização}

No contexto do uso da Internet pelos brasileiros, os dados da Pesquisa Nacional por Amostra de Domicílios Contínua (PNAD) feita pelo Instituto Brasileiro de Geografia e Estatística (IBGE), mostraram que 64,7\% das pessoas, com idade de 10 anos ou mais, utilizaram a Internet no quarto trimestre de 2016. Essa faixa inclui $65,5 \%$ do sexo feminino e 63,8\% do sexo masculino. Quanto aos jovens, com idade entre 18 a 24 anos, foi contabilizada uma porcentagem, significativa, de $85 \%$. Dados semelhantes foram identificados pela pesquisa apresentada nesse trabalho (descrita na seção 4). Por outro lado, pessoas de 60 anos ou mais contabilizaram 25\%. O equipamento mais utilizado pelos usuários para se conectarem, foi o smartphone $(94,6 \%)$, seguido pelo microcomputador $(63,7 \%)$ e tablet $(16,4 \%)$ [IBGE 2018$]$.

Com relação às finalidades de uso da Internet pelos usuários, a pesquisa do IBGE (2018) apontou que: 94,2\% são com o propósito de enviar e receber mensagens de texto, voz ou imagens por aplicativos (excluindo-se o e-mail); 76,4\% assistir a vídeos, inclusive programas, séries e filmes; $73,3 \%$ conversar por chamadas de voz ou vídeo e $69,3 \%$ enviar ou receber e-mails. Tendo em vista que muitas dessas atividades estão presentes em redes sociais, como o Facebook, estudos voltados para a análise do comportamento dos usuários frente às questões de privacidade e segurança dos dados, nesses sistemas, se mostram uma opção justificável.

Além disso, quanto ao nível de instrução (em referência ao nível de escolaridade ou educacional), o estudo apresentado pelo IBGE afirma que a propensão das pessoas a 
utilizarem novas Tecnologias de Informação e Comunicação, aumenta, de forma diretamente proporcional, ao crescimento do nível de instrução (apenas 11,2\% utilizaram a Internet no grupo de pessoas sem instrução). Essa característica é notada nos trabalhos relacionados [Kokolakis 2017] e [Mijuskovic et al. 2015] que são descritos na Seção 3.

Questões relacionadas à segurança e privacidade digital têm chamado a atenção do meio acadêmico-científico e do governo no Brasil. A Comissão Especial de Sistemas de Informação da Sociedade Brasileira de Computação (SBC) prospectou os grandes desafios de pesquisa em Sistemas de Informação no Brasil para o período de 2016 a 2026, e, nesse documento, segurança e privacidade constam como importantes requisitos de alguns dos desafios [Boscarioli et al. 2017].

O governo brasileiro, em 2014, aprovou a Lei No 12.965 - "Marco Civil da Internet" [Lei $\left.\mathrm{N}^{\circ} 12.965,2014\right]$ que estabelece princípios, garantias, direitos e deveres para o uso da Internet no Brasil e abrange três principais aspectos: neutralidade, privacidade e liberdade de expressão. E, recentemente, aprovou a Lei Geral de Proteção de Dados Pessoais (LGPD) que trata, dentre outros, do consentimento de usuários, dos requisitos para tratamento de dados pessoais, e dos direitos para o usuário, como: acesso, correção e eliminação dos dados coletados [Lei Nº 13.709, 2018].

Esse artigo discute as percepções e preferências sobre segurança e privacidade no Brasil e faz uma comparação com outros países no viés do paradoxo da privacidade. $\mathrm{O}$ artigo está assim estruturado: na seção seguinte, discute-se o conceito do Paradoxo da Privacidade, na Seção 3 são apresentados trabalhos que discutem o comportamento e percepção dos usuários frente às questões de privacidade e segurança de dados na Internet; a quarta seção apresenta um projeto desenvolvido pelo grupo e, por fim, uma seção de discussão sobre os resultados alcançados.

\section{Paradoxo da Privacidade}

Em muitas pesquisas realizadas com usuários de redes sociais e aplicativos móveis, esses dizem se preocupar com privacidade. Entretanto, em determinados contextos, suas ações mostram o contrário. $\mathrm{O}$ termo Paradoxo da Privacidade afirma que há divergência entre intenção e comportamento real.

A revisão da literatura feita em Kokolakis (2017) sobre o Paradoxo da Privacidade traz algumas interpretações para tentar explicar a ocorrência desse fenômeno:

- Cálculo de Privacidade - há recompensas intangíveis na divulgação de informações como, por exemplo, ganho de capital social.

- Interpretações baseadas em teorias sociais - consideram questões emocionais, culturais e regras implícitas da sociedade.

- Informação incompleta e assimétrica - falta informações/conhecimento sobre o assunto para o cálculo da privacidade.

- Vieses cognitivos - há limites no processamento de grande quantidade de informações.

- Teoria do Quantum - as preferências dos indivíduos podem mudar na hora de tomar decisões.

Muitas pesquisas tentam comprovar essas questões em especial, sobre a 
perspectiva do cálculo da privacidade, da informação incompleta e assimétrica e do viés cognitivo. Em Silva (2012), artigo de cunho jurídico, o paradoxo da privacidade ocorre "quando a violação da privacidade se dá pela vontade da própria "vítima"." A autora afirma que mesmo com alguns avanços no sentido de resguardar a privacidade, muitos usuários de redes sociais têm buscado propositadamente exibir sua privacidade. Diante desse fato, nesse trabalho, buscou-se sentido para o paradoxo da privacidade frente à obra Lógica do Sentido de Gilles Deleuze. Uma das constatações do artigo é que o sentido em querer se mostrar exibir seu eu para o outro provém da ideia de se criar personagens ou personalidades de si. Esses personagens, investidos geralmente de alguma ludicidade, visam despertar a atenção, conseguir o interesse de outros para si. Por fim, a autora ainda afirma "Não se exibe a privacidade pelo simples desejo de exposição".

Nesse trabalho [Kokolakis 2017], verificou-se que a maioria dos artigos encontrados investigava o paradoxo com técnicas de pesquisa do tipo survey onde se buscava comparar o comportamento real com as declarações dos usuários sobre privacidade. Poucos trabalhos fazem experimentos, comparando a diferença entre o que o respondente pretende divulgar previamente e o comportamento real posterior com coleta efetiva de dados. Mesmo que necessitando de mais recursos talvez esse tipo de pesquisa possa trazer novas contribuições para esta questão paradoxal.

Um modo de se estabelecer a privacidade é por meio da política de privacidade, documento que deve informar ao usuário, quais dados serão solicitados, como será e por quanto tempo armazenados e ainda a finalidade da coleta. Porém, esse tipo de documento é conhecido por seu tamanho excessivo, uso de jargões técnicos, termos vagos e ambiguidade intencional fazendo com o que o usuário não tenha interesse em lê-lo e simplesmente concorde com as condições ora impostas. Essa questão está diretamente relacionada à perspectiva da falta de informação discutida em [Kokolakis 2017].

Por outro lado, se considerarmos o comportamento paradoxal dos usuários, é improvável que as empresas desenvolvedoras de redes sociais e aplicativos se sentissem impelidas a proporcionarem melhores formas de resguardar a privacidade de seus usuários, a não ser pelas questões legais impostas pelo governo [Lei No 12.965 2014; Lei $\left.\mathrm{N}^{0} 13.7092018\right]$.

Por fim, há um dilema imposto ao usuário que de fato se preocupa com sua privacidade, pois ao tempo em que ele precisa usar diversos aplicativos e redes sociais para aumentar sua produtividade, dar agilidade em tarefas diárias bem como se sentir digitalmente sociável, ele está refém em aceitar os termos impostos pela política de privacidade que não fornecem informações completas e coesas sobre a privacidade. Podese afirmar que há certo desequilíbrio entre optar pela comodidade dos serviços digitais ou pela privacidade de seus dados pessoais.

\section{Trabalhos Relacionados}

A pesquisa sobre o comportamento e percepção dos usuários frente às questões de privacidade e segurança de dados na Internet é um tópico que vem sendo muito explorado por vários estudos ao redor do mundo. Nesse sentido, foram encontrados diversos trabalhos com propostas semelhantes a este, aplicados em países com diferentes culturas e crenças no que tange à privacidade e segurança. Nas subseções seguintes são descritos trabalhos correlatos desenvolvidos na Europa, Brasil, e em outros países. 


\subsection{Percepção e Preocupação com a Privacidade e Segurança na Europa}

O trabalho conduzido por Kokolakis (2017) examinou os dados de uma pesquisa com 22.253 pessoas em 26 países da Europa e os dados culturais sobre a privacidade nesses países. Assim, por meio de uma análise, foi gerado um modelo lógico onde são observados os aspectos responsáveis pelo aumento da preocupação dos usuários com a privacidade. Esse modelo indicou que os usuários, de países com índices elevados de preocupação com segurança e desconfiança por organizações, se mostraram mais apreensivos com o uso indevido de suas informações. Somado a isso, a cultura exerceu papel importante nas questões de privacidade e na estabilidade dos padrões, pois valores culturais mudam de forma lenta. A pesquisa também mostrou que países da porção norte e oriental da Europa (Suécia, Finlândia, Estônia, Malta, Hungria) se mostraram menos preocupados com o uso indevido de dados pessoais quando comparados aos países da porção central e sul da Europa (Itália, França, Portugal, Alemanha).

Individualmente, foi notado que a preocupação com o uso indevido de informações pessoais na web é maior entre: 1) indivíduos do sexo feminino e 2) indivíduos do sexo masculino na faixa etária entre 45 a 65 anos, ambos os grupos com níveis de escolaridade elevados. A análise mostra que metodologias com foco na proteção de dados pessoais (como frameworks) podem atuar como campanhas de informação e estimular o aumento da preocupação dos indivíduos em relação à privacidade. Dessa forma, os esforços nacionais, culturais e sócios demográficos foram os responsáveis pela mudança da percepção dos usuários, quanto a sua privacidade na Internet, nos países europeus onde a pesquisa se baseou.

Em Silva (2012) foi utilizado um questionário para medir o grau de conhecimento e consciência dos usuários da Macedônia, quanto à segurança e privacidade de sistemas de nuvem (a exemplo, Google Drive, Dropbox, OneDrive). Um número total de 28 participantes, da área de Tecnologia da Informação, foi incluído nesse estudo. Os resultados mostraram que os usuários, em geral, estão conscientes da existência de riscos quanto à segurança e privacidade ao armazenar dados na nuvem, porém, não possuem conhecimento sobre os tipos de riscos e ameaças, pois apenas $14 \%$ conseguiram discorrer sobre o tema.

Em Cerere et al. (2015), os autores coletaram informações, por meio de um questionário, sobre os usuários de sistemas de Tecnologia da Informação e Comunicação (TIC) em diferentes regiões na Croácia a fim de examinar a situação do país quanto ao nível de percepção e comportamento dos usuários frente aos riscos de segurança e privacidade. A pesquisa contou com a participação de usuários com diferentes níveis de conhecimento, experiência, idade e gênero, totalizando 701 participantes. Pode-se concluir que:

- Os usuários do sexo feminino, geralmente, são mais cautelosos quanto à privacidade e segurança na web em relação ao sexo masculino;

- Os usuários de TIC de meia idade e mais avançados obtiveram resultados positivos no total e na maioria das escalas;

- Os usuários de TIC que trabalham no setor privado revelam suas senhas mais frequentemente.

A comparação de usuários com diferentes qualificações profissionais mostrou que os participantes com mestrado obtiveram resultados significativamente melhores que os 
outros participantes.

\subsection{Percepção e Preocupação com a Privacidade e Segurança no Brasil}

O trabalho Mijuskovic et al. (2015) apresenta uma pesquisa com o objetivo de identificar o grau de preocupação e percepção dos usuários da Internet no Brasil. A coleta de dados foi feita por meio de um questionário, distribuído nas cinco regiões do país (Sul, Sudeste, Centro-Oeste, Norte e Nordeste), totalizando 1104 respostas com foco no comportamento do usuário e preocupação com a privacidade. Os resultados indicaram quanto ao perfil dos usuários que a maioria dos respondentes é do sexo feminino $(61,8 \%)$ e a faixa etária é de 25 a 35 anos $(46,3 \%)$.

Quanto à ocupação desses participantes, os resultados mostraram que 51,4\% estão empregados; $13,2 \%$ são estudantes sem atividade profissional e 12,8\% marcaram a opção "outro" (aposentados, desempregados ou estagiários). Finalmente, quanto à preocupação com a segurança das informações:

- 822 respostas indicaram uma forte preocupação com as informações de senhas $(74 \%)$

- 806 com número de cartão de crédito (73\%);

- 740 com número de conta corrente e agência (67\%);

- 712 com saldo bancário (64\%);

- 696 com gastos com cartão de crédito (63\%).

O sexo masculino demonstrou, entre todos os campos, maior preocupação com as informações de senhas $(86,02 \%)$ e o sexo feminino indicou uma maior preocupação com relação ao número de cartão de crédito $(87,54 \%)$. A informação indicada como menos sensível, por ambos os sexos, foi a orientação sexual. Os resultados do trabalho servem como reflexão aos usuários de Internet no Brasil sobre a importância da privacidade e segurança das informações na Internet.

\subsection{Percepção e Preocupação com a Privacidade e Segurança em Outros Países}

O estudo Thomson et al. (2015) compara os usuários de redes sociais do Japão com os Estados Unidos e propõe um modelo teórico a partir dos conceitos sócios ecológicos (que relaciona as características de um ambiente como interdependentes), mobilidade relacional e a confiança geral na questão da privacidade. Considerando-se que a mobilidade relacional no Japão é baixa (a confiança geral em estranhos é, geralmente, baixa), o estudo levantou como hipótese a preocupação com a privacidade ser elevada. Em contraste a isso, nos Estados Unidos, que apresenta alta mobilidade relacional, a confiança geral também é elevada, levando a previsão de um índice de preocupação com privacidade menor.

Essas hipóteses foram testadas e os resultados mostraram que o Japão, como estimado, apresentou números maiores quanto à preocupação com a privacidade em detrimento dos Estados Unidos. As descobertas desse estudo sustentam a importância de levar em consideração os fatores sócios ecológicos off-line ao abordar as diferenças interculturais na privacidade em relação à Internet.

Em um outro estudo, Guzman et al. (2010), consistiu em uma pesquisa 
social/comportamental sobre práticas de segurança da informação pelos usuários de internet na Bolívia. O modelo construído utiliza a teoria cognitiva social para explicar os fatores individuais que influenciam no comportamento de segurança da informação. Esse estudo aborda questões como a eficiência da segurança da informação influenciando o comportamento de segurança da informação; a conscientização sobre segurança como ferramenta para influenciar o comportamento de segurança da informação e as diferenças comportamentais quanto à segurança da informação por meio de fatores educacionais, gênero, frequência de uso do computador e Internet.

Essa pesquisa contou com 255 participantes. Os resultados mostraram que os usuários de internet na Bolívia estão conscientes dos problemas de segurança comuns, como a necessidade de utilizar antivírus. No entanto, existe pouco conhecimento sobre políticas de segurança, e isso se deve ao fato de que muitas organizações não seguem práticas formais de gerenciamento de segurança [Guzman et al. 2010].

\section{Projeto "Cartilha de Segurança e Privacidade Digital"}

Como foi apresentado nos trabalhos relacionados [Kokolakis 2017], [Silva 2012], [Cerere et al. 2015], [Mijuskovic et al. 2015], [Thomson et al. 2015] e [Guzman et al. 2010], o esforço em promover uma conscientização dos usuários da Internet sobre questões de segurança e privacidade, tem sido uma solução comum na forma em lidar com problemas referentes a proteção de dados sensíveis. Nesse sentido, em 2017 iniciou-se um projeto de extensão no Instituto de Computação da UFMT (Universidade Federal de Mato Grosso) sobre conscientização e sensibilização sobre os riscos na Web intitulado "Cartilha de Segurança e Privacidade Digital”.

O principal objetivo do projeto era ser um canal de divulgação de informações sobre os riscos inerentes a segurança e privacidade digital dos usuários da Internet ao mesmo tempo em que se pretendia levantar um perfil simplificado do comportamento dos usuários da Internet na Universidade Federal de Mato Grosso quanto a algumas competências relativas à segurança e privacidade digital, tais como: leitura de termo de uso e política de privacidade, troca regular de senhas, comportamento seguro na Web. Contudo, a escolha da rede social Facebook como ferramenta de divulgação do projeto necessitou uma mudança no grupo avaliado, tendo que ampliar da UFMT para a sociedade de modo geral, uma vez que ficaria impossibilitado de limitar a pesquisa realizada por meio de questionário eletrônico postado na Fanpage do projeto.

A metodologia do projeto envolveu o estudo contínuo acerca de novas tipificações de crimes virtuais, invasão de privacidade, coleta, armazenamento e distribuição de dados privados bem como formas de se proteger. Para tanto, foi necessário acompanhar páginas de notícias sobre tecnologia, bem como leitura de artigos científicos sobre o tema do projeto. Para a elaboração dos materiais disponibilizados no Facebook, foram utilizados sistemas gratuitos para a postagem de artigos, vídeos, figuras, questionários. A escolha pelo Facebook como ferramenta de divulgação da cartilha digital deu-se, principalmente, por ser uma rede social online com grande abrangência de usuários, em especial no Brasil. A página da fanpage do projeto pode ser encontrada em https://www.facebook.com/lavisecandpriv.

Outro ponto importante da metodologia foi a aplicação de um questionário relacionado ao comportamento dos usuários com relação a segurança e privacidade digital onde foram agrupados os participantes em quatro categorias: com relação ao seu vínculo 
com a UFMT: alunos, professores, técnicos e público externo. O questionário continha oito questões objetivas e discursivas e obteve 128 respostas no período de 26/09/2017 até $23 / 10 / 2017$.

Com base nas respostas do questionário referente a proteção de dados sensíveis na Internet, identificou-se que $92,2 \%$ dos participantes responderam que se preocupam com a segurança e a privacidade dos seus dados na Internet. Enquanto 4,7\% afirmaram não se preocupar.

Com relação a leitura dos termos de uso e/ou a política de privacidade antes de instalar um aplicativo, aproximadamente $85,1 \%$ afirmaram que nunca ou raramente ou somente às vezes leem os termos de uso/política de privacidade. Em contrapartida 14,9\% afirmou que na maioria das vezes ou sempre leem os termos de uso e/ou a política de privacidade.

A resposta ao item sobre o costume dos participantes em trocar suas senhas em aplicativos e redes sociais, 55,5\% afirmaram que nunca ou raramente trocam suas senhas. Por outro lado, um conjunto de participantes respondeu que $22,7 \%$ trocam suas senhas, mas somente quando são notificados e $21,9 \%$ periodicamente trocam suas senhas.

Os participantes afirmaram na questão sobre quais categorias ocorrem associação com seu perfil de usuário em rede social que $60,2 \%$ raramente postam alguma coisa, usando a rede social mais para ler o que os outros postam nela, $32 \%$ divulgam aquilo que acham interessante, 32\% também divulgam o que acham interessante, mas sempre verifica a veracidade, $4,7 \%$ postam fotos sem se preocupar com quem vê, $1,6 \%$ escrevem tudo o que vem à cabeça e 5,6\% deram outras respostas.

Quando perguntados sobre a visão deles sobre a questão da privacidade hoje, em uma sociedade conectada pelas tecnologias, os participantes responderam na forma dissertativa e na tabulação das respostas houve uma sintetização em quatro afirmações principais: "Fraca, sem legislação e controle", "Privacidade é algo difícil de alcançar", "A privacidade é importante" e "Privacidade não existe".

Das pessoas que participaram da pesquisa, a grande maioria são jovens e adultos onde $53,9 \%$ pertencem a faixa etária de 21 a 30 , seguido de $20,3 \%$ pertencente a faixa etária de 31 a 40 e 14,1\% pertencente a faixa etária de 41 a 50 . Tendo apenas 4,7\% pertence a faixa etária de 16 a 20, também outros $7 \%$ de respostas pertencentes a faixa etária de 51 a 70. Com relação ao sexo dos participantes, 72,7\% são masculinos e 27,3\% feminino.

Sobre o vínculo do participante com a UFMT, 46,9\% são estudantes, seguidos de $30,5 \%$ que representam pessoas que não possuem vínculo com a UFMT, tendo ainda $14,8 \%$ de professores e ainda $7,8 \%$ que correspondem aos técnicos.

$\mathrm{Na}$ próxima seção é realizada uma análise mais aprofundada dos dados apresentados no questionário aplicado no projeto.

\section{Discussão e Consolidação dos Resultados Alcançados}

Pode-se perceber que os resultados da pesquisa obtidos no projeto "Cartilha de Segurança e Privacidade Digital", apesar da pequena abrangência, são consistentes com a hipótese do Paradoxo da Privacidade. Nota-se que na opinião de participantes que responderam possuir preocupação com a segurança e privacidade de seus dados na Internet, a maior 
parte deles afirmou que nunca leem, raramente leem ou somente às vezes leem os termos de uso ou a política de privacidade. Dessa forma, percebe-se que os fatores socioecológicos [Thomson et al. 2015] também influenciam o grupo pesquisado, uma vez que os brasileiros como os americanos também possuem alta mobilidade relacional, propiciando uma confiança geral elevada e um índice de preocupação com privacidade baixo.

Essa influência é corroborada pela Tabela 1 que apresenta dados de uma pesquisa realizada em Thomson et al. (2015) onde mostra o prejuízo financeiro gerado por crimes cibernéticos em 2017. Percebe-se semelhança entre Brasil e EUA com relação ao alto prejuízo financeiro, em contraponto ao valor apresentado no Japão. Isso reforça que uma confiança elevada pode gerar uma menor preocupação com a privacidade, podendo ocasionar perdas financeiras consideráveis.

\section{Tabela 1. Prejuízo Financeiro Gerado por Crimes Cibernéticos em 2017}

\begin{tabular}{|c|c|c|c|}
\hline Ano & Brasil & EUA & Japão \\
\hline 2017 & US\$ $22.5 \mathrm{~B}$ & US\$ $19.4 \mathrm{~B}$ & US\$ $2.1 \mathrm{~B}$ \\
\hline
\end{tabular}

Em relação aos participantes que responderam ter preocupação com a segurança e a privacidade dos seus dados que circulam na Internet, com o costume deles em trocar suas senhas em aplicativos e redes sociais, pode-se analisar que a maior parte dos participantes afirmou se preocupar com a segurança de seus dados na Internet, mas a maioria reconheceu que raramente troca a senha, somente quando é notificado pelo sistema/aplicativo ou nunca troca sua senha.

Considerando as devidas proporções, em Thomson et al. (2015) há uma certa convergência com os dados apresentados no projeto, quando afirma que $1 / 3$ dos entrevistados armazenam de forma insegura suas senhas e $20 \%$ deles usam a mesma senha em todas suas contas.

O projeto tem buscado ampliar sua linha de atuação, extrapolando a Fan Page, lançando uma segunda plataforma de divulgação no Instagram, ministrando palestras e oficinas em eventos acadêmicos e corporativos, desenvolvendo novos estudos e publicando os resultados em eventos científicos e nas próprias redes sociais.

Recentemente, em 2019, foi realizada uma nova pesquisa com alunos dos cursos de ciência da computação e de sistemas de informação da UFMT quanto à segurança e a privacidade de seus dados no meio digital [Vittorazi et al. 2019]. Apesar da hipótese inicial do trabalho partir do pressuposto de que alunos de cursos da área de tecnologia teriam um comportamento mais cuidadoso no acesso à Internet devido terem mais acesso a informações sobre os riscos de segurança e privacidade na Web, o que se observou foi um contraponto.

Os dados mostraram que muitos estudantes têm um comportamento displicente em relação à segurança de seus dados: não têm o costume de trocar senha, não leem os termos de uso antes de instalar um aplicativo e somente a minoria dos respondentes $(12 \%)$ afirmaram saber configurar os parâmetros de segurança e privacidade em aplicativos. Além disso, ficou comprovado que os estudantes tinham conhecimento superficial do 
Marco Civil da Internet [Lei N $\left.{ }^{0} 12.965,2014\right]$ bem como da Lei Geral de Proteção de Dados (LGPD) [Lei N ${ }^{\circ}$ 13.709, 2018].

De modo preliminar, essa amostra pesquisada, sugere que pessoas com conhecimento formal de computação não estão livres de terem comportamento de risco na Web e poucas delas sabem contramedidas para se proteger de possíveis ataques por meio de aplicativos.

As duas pesquisas exploratórias, mesmo que tendo perfis de amostragem por conveniência diferentes, demonstram certa correlação e corroboram com o fato de que é necessário investir na educação digital, em especial, nos temas de segurança e privacidade digital.

O projeto continua criando materiais instrucionais buscando proporcionar uma educação digital sobre segurança e privacidade digital aos usuários que acompanham o projeto nas redes sociais e, iniciou um trabalho junto a disciplina de Tecnologia e Sociedade onde os próprios alunos são convidados a pesquisar a temática e criar materiais digitais, fazendo assim um elo entre a disciplina e o projeto de extensão bem como colocando os alunos como agentes ativos de seu aprendizado.

Em trabalhos futuros pretende-se executar pesquisas exploratórias na área de proteção de dados, uma vez que esse tema está se tornando uma importante linha de pesquisa, devido a entrada em vigor da Lei Geral de Proteção de Dados Brasileira (em 2021) e a Lei Geral de Proteção de Dados Europeia (em 2019). Contudo, com a mesma finalidade apresentada neste trabalho, incentivar o comportamento seguro, privado e ético na Internet por meio de materiais (cartilhas, postagens, podcasts, vídeos) de conscientização e sensibilização nas mídias sociais.

\section{Referências}

Acquisti, A. (2004). Privacy in electronic commerce and the economics of immediate gratification. in: Proceedings of the 5th ACM conference on Electronic commerce, $p$. 21-29.

Barnes, S. B. (2006). "A privacy paradox: Social networking in the United States". First Monday, 11(9).

Boscarioli, C., De Araujo, R. M., \& Maciel, R. S. P. (2017). I GranDSI-BR Grand Research Challenges in Information Systems in Brazil 2016-2026.

Cecere, G., Le Guel, F., \& Soulié, N. (2015). Perceived Internet privacy concerns on social networks in Europe. Technological Forecasting and Social Change, 96, p. 277287.

Guzman, I. R., Galvez, S. M., Stanton, J. M., \& Stam, K. R. (2010). Information Security Practices in Latin America: The case of Bolivia. In AMCIS, p. 492.

IBGE. (2018) "Pesquisa Nacional por Amostra de Domicílios Contínua - Acesso à Internet e à televisão e posse de telefone móvel celular para uso pessoal 2016", www.agenciadenoticias.ibge.gov.br/agencia-sala-de-imprensa/2013-agencia-denoticias/releases/20073-pnad-continua-tic-2016-94-2-das-pessoas-que-utilizaram-ainternet-o-fizeram-para-trocar-mensagens.html.

Kokolakis, S. (2017). Privacy attitudes and privacy behaviour: A review of current 
research on the privacy paradox phenomenon. Computers \& Security, 64, p. 122-134.

Lei $\quad \mathrm{N}^{\mathrm{O}} \quad 12.965 \quad$ (2014) www.planalto.gov.br/CCIVIL_03/Ato20112014/2014/Lei/L12965.htm.

Lei $\quad \mathrm{N}^{\mathrm{O}} \quad 13.709 \quad$ (2018) http://www.planalto.gov.br/ccivil_03/_ato20152018/2018/lei/L13709.htm.

Mijuskovic, A., \& Ferati, M. (2015). User awareness of existing privacy and security risks when storing data in the cloud. In International Conference on e-Learning and the Knowledge Society, European Commission, p. 268-273

Norton (2017) "Norton Cyber Security Insights Report", us.norton.com/cyber-securityinsights-2017.

Silva, V. R. B. D. (2015). Preocupação com a privacidade na internet: uma pesquisa exploratória no cenário brasileiro, Anais do V Encontro de Administração da Informação, 2015, Brasil.

Solic, K., Velki, T., e Galba, T. (2015). Empirical study on ICT system's users' risky behavior and security awareness. In 2015 38th International Convention on Information and Communication Technology, Electronics and Microelectronics (MIPRO), p. 1356-1359. IEEE.

Thomson, R., Yuki, M., \& Ito, N. (2015). A socio-ecological approach to national differences in online privacy concern: The role of relational mobility and trust. Computers in Human Behavior, 51, p. 285-292.

Vittorazi, D., Araujo, N., Souza, P. (2019). Investigando o Comportamento na Web de um Grupo de Bacharelandos da Área de Tecnologia da Informação. Anais ERI-MT, Cuiabá, 2019. 